\title{
State Transition, Balancing, Station Keeping, and Yaw Control for a Dynamically Stable Single Spherical Wheel Mobile Robot
}

\author{
Umashankar Nagarajan, Anish Mampetta, George A. Kantor and Ralph L. Hollis
}

\begin{abstract}
Unlike statically stable wheeled mobile robots, dynamically stable mobile robots can have higher centers of gravity, smaller bases of support and can be tall and thin resembling the shape of an adult human. This paper concerns the ballbot mobile robot, which balances dynamically on a single spherical wheel. The ballbot is omni-directional and can also rotate about its vertical axis (yaw motion). It uses a triad of legs to remain statically stable when powered off. This paper presents the evolved design with a four-motor inverse mouseball drive, yaw drive, leg drive, control system, and results including dynamic balancing, station keeping, yaw motion while balancing, and automatic transition between statically stable and dynamically stable states.
\end{abstract}

\section{INTRODUCTION}

Design and control of dynamically stable mobile robots is a growing area of research. Conventional statically stable mobile robots generally have large and heavy bases to ensure low centers of gravity, making them ill-suited for navigation in human environments. Robots for operation in human environments ideally should be tall and thin. These attributes can best be achieved with dynamic stability.

One of the first two-wheeled inverse pendulum type mobile robot was designed and controlled in [1]. The Segway RMP two-wheeled self-balancing mobile robot base has been a popular platform in recent years [2]. Very capable twowheeled mobile manipulators have been introduced [3]. Such two-wheeled balancing platforms have dominated the field of dynamically stable mobile robots but they have kinematic constraints that restrict their direction of motion. In 2005, the authors' group introduced the ballbot, a dynamically stable mobile robot moving on a single spherical wheel [4], [5], and popularized in [6]. The single spherical wheel provides omnidirectional motion making the ballbot more suitable for navigation in human environments with constrained spaces. Moreover, unlike its two-wheeled counterparts, the ballbot is as tall as an adult human, which enhances its ability to interact with human environments. Recently, others have developed similar robots [7], [8].

This paper presents an evolved version of the ballbot with a four-motor inverse mouse-ball drive, a yaw drive that provides rotation about its vertical axis and a leg drive that enables controlled transition from statically stable state (SSS) to dynamically stable state (DSS) and vice versa. Design details are given in Sec. II. Section III provides a simplified

This work is supported by NSF Grants IIS-0308067 and IIS-0535183.

The authors are with The Robotics Institute, Carnegie Mellon University, Pittsburgh, PA 15213, USA umashankar@cmu.edu, kantoreri.cmu.edu and rhollisecs.cmu.edu

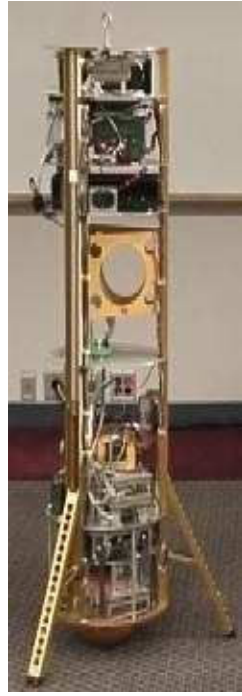

(a)

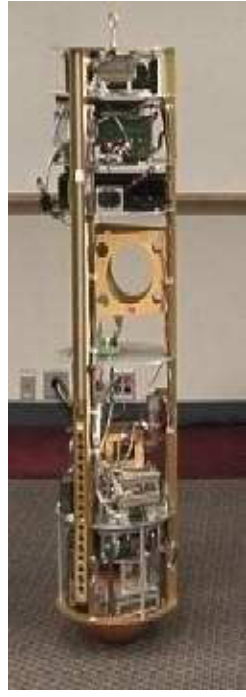

(b)
Fig. 1. Ballbot: (a) statically stable, $(b)$ dynamically stable.

ballbot dynamic model, with parameter measurements presented in Sec. IV. Section V describes an improved, robust balancing and station keeping controller, yaw controller, leg controller and an automatic procedure for transition between SSS and DSS and vice versa. Sections VI and VII present the results and conclusions.

\section{SYSTEM DESCRIPTION}

\section{A. Four-Motor Inverse Mouse-Ball Drive}

The previous version of the inverse mouse-ball drive [5], had a pair of drive and opposing passive rollers for each of the orthogonal motion directions. With this scheme, the drive rollers produced an upward or downward force on the urethane-covered ball in addition to torque. This setup, while operable, resulted in unequal friction for forward and backward motion in addition to some undesirable "hopping motion." To avoid this, the present design has all four rollers actuated with individual DC servomotors which exert pure torques on the ball. Figure 2(a) illustrates the main features of this arrangement.

\section{B. Yaw Drive}

The ball drive mechanism is attached to the body using a large thin-section bearing which allows yaw rotation. The yaw drive shown in Fig. 2(b) has a DC servomotor with planetary gears driving the pulley assembly at the center. An absolute encoder attached to the pulley assembly gives 


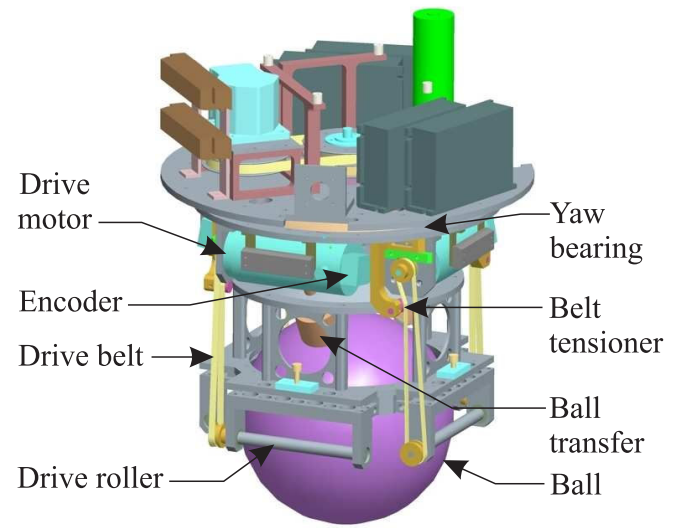

(a)

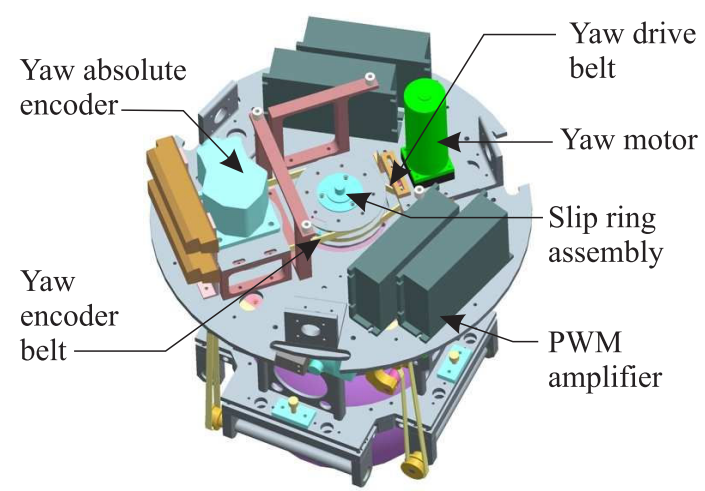

(b)

Fig. 2. Four-motor inverse mouse-ball drive and yaw drive: (a) view showing main drive arrangement, $(b)$ view showing yaw drive mechanism.

the orientation of the body frame with respect to the ball drive unit. A slip ring assembly for drive motor currents and encoder signals permits unlimited yaw rotation.

\section{Leg Drive}

Fig. 3 shows snapshots of the leg drive mechanism. Each $0.48 \mathrm{~m} \mathrm{leg}$ is driven by a linear screw drive with a ratio of $1.6 \mathrm{~mm} / \mathrm{revolution}$. The tip of the leg has a hoof switch and a ball castor. The hoof switch indicates contact with the floor and the ball caster allows some horizontal rolling motion along the floor. A spring and linear guide between the leg and the hoof adds some compliance. The legs are independently driven by three DC servomotors with $500 \mathrm{cpr}$ encoders.

\section{PLANAR SIMPLIFIED BALLBOT MODEL}

The ballbot is modeled as a rigid cylinder on top of a rigid sphere. The ballbot dynamics are simulated and control strategies are tested using this planar model. The following assumptions are made: $(i)$ there is no slip between the ball and the floor, (ii) motion in the median sagittal plane and median coronal plane is decoupled, and (iii) the equations of motion in these two planes are identical. With these assumptions a pair of decoupled, independent controllers that stabilize the 3D system can be designed.

Euler-Lagrange equations are used to derive the dynamic equations of motion of the planar ballbot model shown in

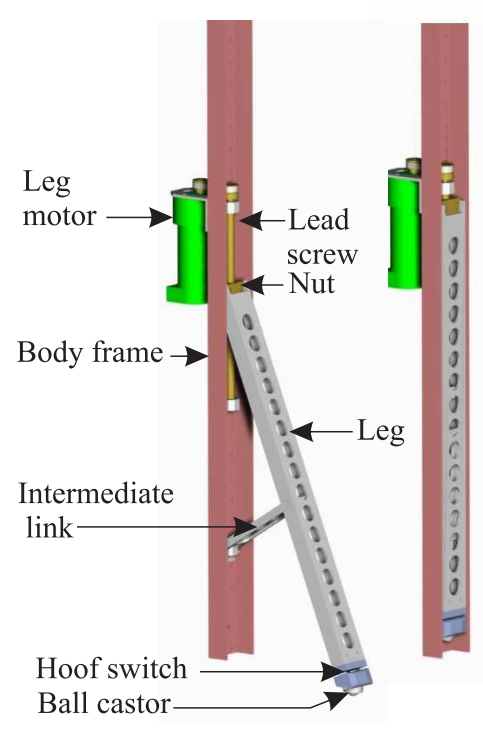

(a)

(b)

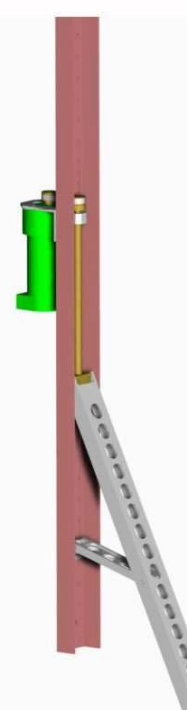

(c)
Fig. 3. Leg drive: (a) Various components of the leg drive mechanism, $(b)$ legs completed retracted, and (c) legs completely deployed.

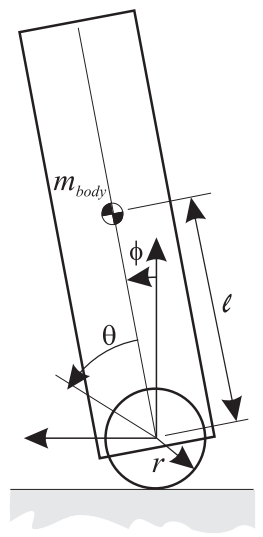

Fig. 4. Planar simplified ballbot model.

Fig. 4. It is to be noted that the model described below uses a coordinate scheme different from the one described in [5]. The coordinate scheme shown in Fig. 4 avoids the coupling between the two generalized coordinates unlike the coordinate scheme in [5]. Moreover, the coordinate scheme described here give direct sensor outputs on the real robot. The generalized coordinate vector of the system is defined as $q=[\theta, \phi]^{T}$. The Euler-Lagrange equations of motion for the simplified planar ballbot model are:

$$
\frac{d}{d t} \frac{\partial L}{\partial \dot{q}}-\frac{\partial L}{\partial q}=\left[\begin{array}{l}
\tau \\
0
\end{array}\right]-\left[\begin{array}{c}
D_{c} \operatorname{sgn}(\dot{\theta})+D_{v} \dot{\theta} \\
0
\end{array}\right],
$$

where $L$ is the Lagrangian, $\tau$ is the torque applied between the ball and the body in the direction normal to the plane, $D_{c}$ is the coulomb friction and $D_{v}$ is the viscous damping friction between the ball and the four rollers, 3 support balls and the ground as well as the motor back-EMFs. The equations of motion can be re-written in matrix form as follows:

$$
M(q)\left[\begin{array}{c}
\ddot{\theta} \\
\ddot{\phi}
\end{array}\right]+C(q, \dot{q})+G(q)+D(\dot{q})=\left[\begin{array}{l}
\tau \\
0
\end{array}\right],
$$


where $M(q)$ is the mass matrix, $C(q, \dot{q})$ is the vector of coriolis and centrifugal forces and $G(q)$ is the vector of gravitational forces.

The mass matrix is given by:

$$
M(q)=\left[\begin{array}{cc}
\alpha & \alpha+\beta \cos \phi \\
\alpha+\beta \cos \phi & \alpha+\gamma+2 \beta \cos \phi
\end{array}\right],
$$

where $\alpha=I_{\text {ball }}+\left(m_{\text {ball }}+m_{\text {body }}\right) r^{2}, \beta=m_{\text {body }} r \ell$ and $\gamma=$ $I_{\text {body }}+m_{\text {body }} \ell^{2}$. I and $m$ refer to the moment of inertia and mass of the subscripted components respectively, while, $r$, $\ell$ and $g$ are the radius of the ball, position of the center of mass of the body along the vertical axis, and acceleration due to gravity, respectively.

The vector of coriolis and centrifugal forces is given by:

$$
C(q, \dot{q})=\left[\begin{array}{l}
-\beta \dot{\phi}^{2} \sin \phi \\
-\beta \dot{\phi}^{2} \sin \phi
\end{array}\right]
$$

and the vector of gravitational forces is given by:

$$
G(q)=\left[\begin{array}{c}
0 \\
-\frac{\beta g \sin \phi}{r}
\end{array}\right] .
$$

The state vector is defined to be $x=\left[q^{T} \dot{q}^{T}\right]^{T}$ and the input is defined to be $u=\tau$. Therefore, Eq. (2) can be re-written of the form $\dot{x}=f(x, u)$.

TABLE I

SYSTEM PARAMETERS

\begin{tabular}{|l|l|l|}
\hline Parameter & Symbol & Value \\
\hline \hline Z-axis CM from ball center & $l$ & $0.69 \mathrm{~m}$ \\
\hline Ball radius & $r$ & $0.106 \mathrm{~m}$ \\
\hline Ball mass & $m_{\text {ball }}$ & $2.44 \mathrm{~kg}$ \\
\hline Ball inertia & $I_{\text {ball }}$ & $0.0174 \mathrm{kgm}^{2}$ \\
\hline Roll moment of inertia about CM & $I_{x x}^{c m}$ & $12.59 \mathrm{kgm}^{2}$ \\
\hline Pitch moment of inertia about CM & $I_{y y}^{c m}$ & $12.48 \mathrm{kgm}^{2}$ \\
\hline Yaw moment of inertia about CM & $I_{z z}^{c m}$ & $0.66 \mathrm{kgm}^{2}$ \\
\hline Body mass & $m_{\text {body }}$ & $51.66 \mathrm{~kg}$ \\
\hline Coulomb friction torque & $D_{c}$ & $4.39 \mathrm{Nm}$ \\
\hline Viscous damping friction coefficient & $D_{v}$ & $0.17 \mathrm{Nms} / \mathrm{rad}$ \\
\hline
\end{tabular}

\section{PARAMETER ESTIMATION EXPERIMENTS}

To instantiate the mathematical model, off-line experiments were conducted to determine principal system parameters.

\section{A. Inertia Measurement}

Moments of inertia of the body were experimentally determined using a torsional pendulum setup [9]. The ballbot's body was suspended about its center of mass using a torsional spring as shown in Fig. 5 and the oscillations were observed after an initial disturbance. The body angles were recorded using the IMU to find the frequency of oscillations as shown in Fig. 6. The torsional spring constant was obtained by performing the same experiment with an I-beam whose inertia was known. The torsional spring constant is given by

$$
K=I \omega_{n}^{2},
$$

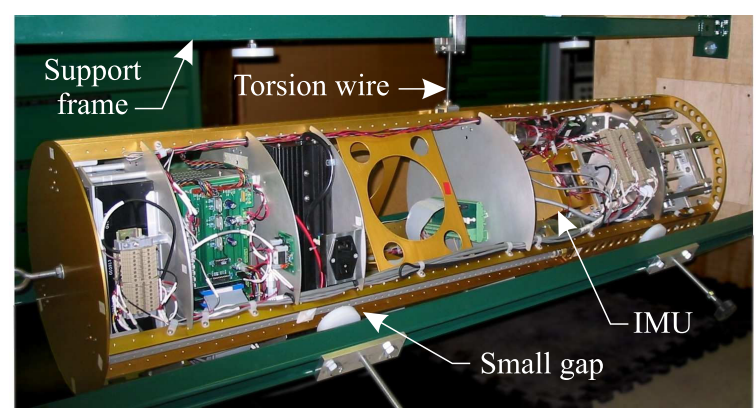

Fig. 5. Torsional pendulum setup with ballbot suspended perpendicular to its length.

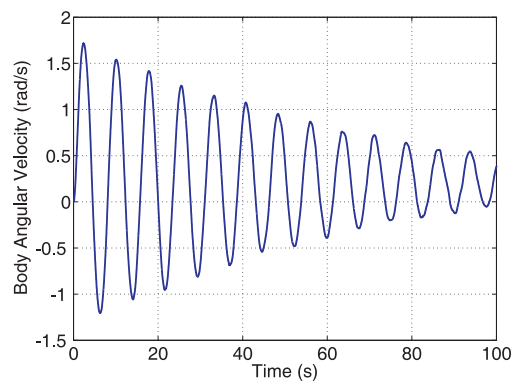

Fig. 6. Damped sinusoidal oscillation used to determine the ballbot's moments of inertia.

where $I$ is the inertia of the suspended object and $\omega_{n}$ is the natural frequency of oscillations. Therefore, the moment of inertia of the body about its center of mass is given by

$$
I_{\text {body }}^{c m}=I_{I-\text { beam }}^{c m} \frac{\omega_{I-\text { beam }}^{2}}{\omega_{\text {body }}^{2}} .
$$

Similar experiments determined $I_{z z}^{c m}$ about the vertical axis by hanging the ballbot vertically. The measured inertia values are shown in Table I. The moments of inertia of the body $I_{x x}$ and $I_{y y}$ about the center of the ball were computed using the parallel-axis theorem.

\section{B. Friction Modeling}

The coulomb and viscous friction terms were determined experimentally by standing the ballbot on a roller ball with its body constrained vertically as shown in Fig. 7. A ramp current (torque) input was given to the ball while recording the angular velocity of the ball. The experiment was repeated with the torque vector at $5^{\circ}$ intervals. A polar plot of the breakaway current is shown in Fig. 8. The average breakaway current provided the value of coulomb friction. The plot of

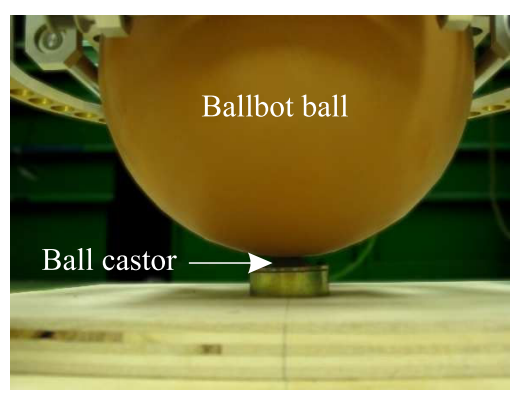

Fig. 7. Ball rolling on the roller during friction tests, 


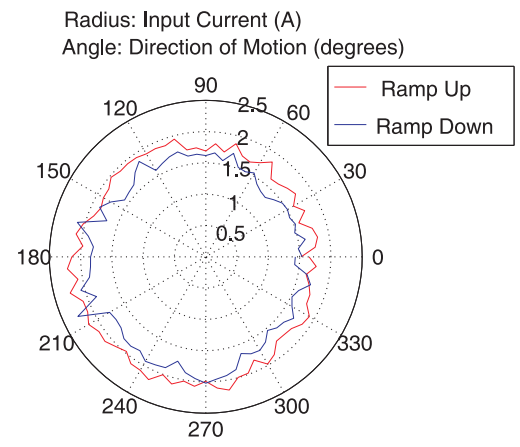

Fig. 8. Polar plot of the breakaway current in different directions of motion.

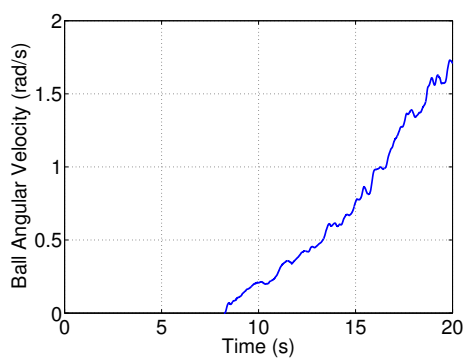

Fig. 9. Linear variation of the ball velocity after breakaway

velocity vs. time after breakaway shown in Fig. 9 can be approximated by a line of constant slope for small speeds. The slopes of the velocity plot and the ramp input were used to determine the viscous friction [10] as shown in Table I.

\section{BALLBOT CONTROL}

\section{A. Balancing}

Balancing for the 3D ballbot system uses two independent controllers operating one in each of the vertical planes. These controllers attempt to move the center of the ball directly below the body center of mass. Each is a ProportionalIntegral-Derivative (PID) controller whose gains were tuned manually. The control system block diagram is shown in the shaded part of Fig. 10. The balancing controller takes the desired body angle $\phi_{d}$ as input (zero for standing still) and tries to balance about that angle. One can feed in desired angles to the balancing controller to move the ballbot around as shown in a companion paper [11].

\section{B. Station Keeping}

The balancing controller is good at balancing about zero body angles but does not bother about the ball's position on

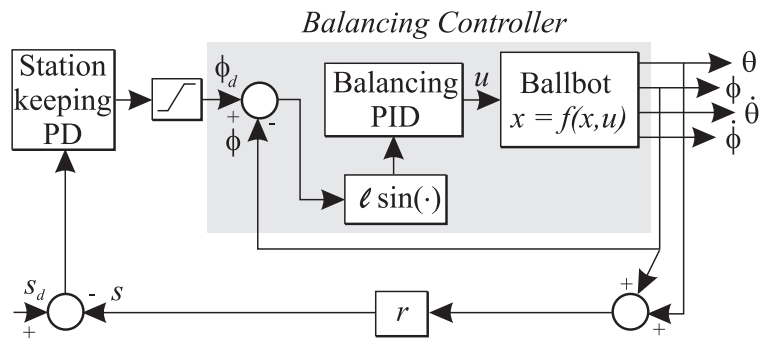

Fig. 10. Block diagram for the station keeping controller with the balancing control block.

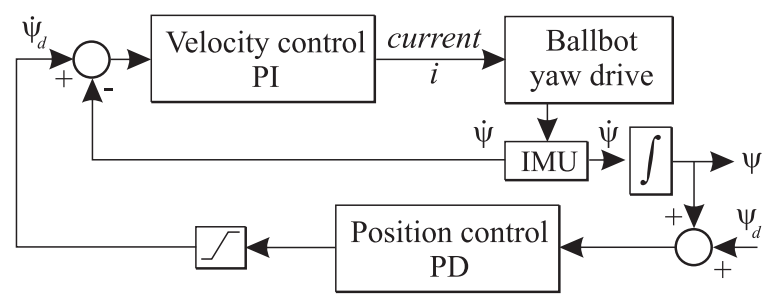

Fig. 11. Block diagram of the yaw controller.

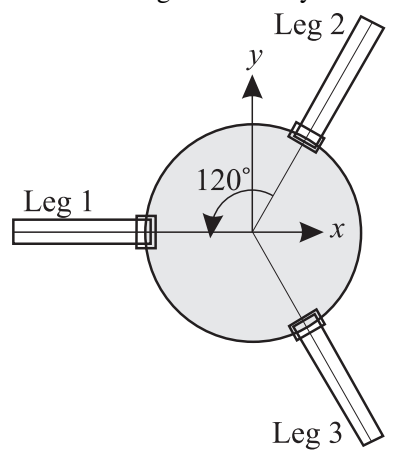

Fig. 12. Top view of the ballbot with all three legs deployed.

the floor. Station keeping is the act of balancing at a desired position even when disturbed. The station keeping controller is achieved with an outer loop around the balancing controller as shown in Fig. 10. It is a Proportional-Derivative (PD) controller that outputs desired body angles depending on the error between the ball's current and desired positions. The angle output from the PD controller is saturated to avoid large lean angles. Gains were tuned manually. This controller replaces the LQR controller of [5].

\section{Yaw Control}

The yaw drive mechanism has an independent controller decoupled from the balancing control for simplicity and ease of control, as shown in Fig. 11. There are two loops: an inner Proportional-Integral (PI) control loop that feeds back the yaw angular velocity $\dot{\psi}$ and an outer PD control loop that feeds back both the yaw angle $\psi$ and yaw angular velocity. The desired angular velocity output from the PD controller is saturated to avoid high angular velocities which could potentially drive the system unstable while balancing.

During yaw motion, the IMU attached to the body frame rotates while the ball drive unit does not. The angle offset $\chi$ between the drive unit and the body frame is given by the absolute yaw encoder. This requires a rotation transformation to be performed to obtain the roll and pitch angles about the ball drive coordinate frame as shown below:

$$
\left[\begin{array}{c}
\text { roll }_{\text {drive }} \\
\text { pitch }_{\text {drive }}
\end{array}\right]=\left[\begin{array}{cc}
\cos \chi & -\sin \chi \\
\sin \chi & \cos \chi
\end{array}\right]\left[\begin{array}{c}
\text { roll }_{\text {body }} \\
\text { pitch }_{\text {body }}
\end{array}\right] \text {. }
$$

D. Leg control

The three legs have independent controllers for lifting the legs up and putting them down. The legs-up controller is a PI speed controller that stops when the legs hit the body, i.e. when the leg speed is less than a defined low threshold speed. The legs-down controller has an inner PI control loop 


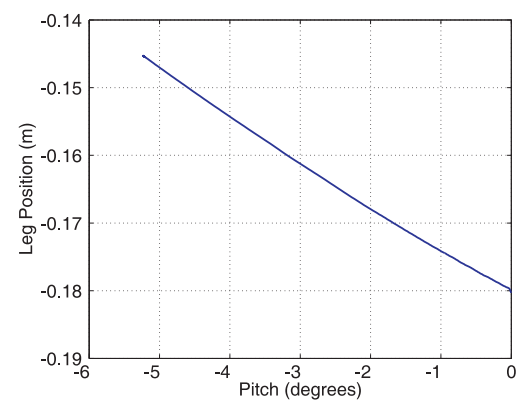

Fig. 13. Position of leg 1 as a function of body pitch.

that feeds back the leg speed and an outer PD control loop that feeds back both leg position and leg speed similar to that of the yaw controller.

In terms of stability, Ballbot has two states: (i) Dynamically Stable State (DSS), in which it balances on top of the ball and (ii) Statically Stable State (SSS), in which it rests with all the three legs deployed. Motion is possible in both the modes [12]. In DSS, ballbot moves around by balancing on the ball and leaning the body in the desired direction of motion. while in SSS, the robot moves by rolling the ball with all the three legs sliding on the floor. However, in SSS, motion is restricted to smooth planar surfaces.

For the ballbot to be fully autonomous, it must be capable of automatic transition between the two states. When the legs are down, the ballbot may not be exactly vertical. To transition from the SSS to DSS state, the balancing controller and legs-up controller must operate together, which can create large transients. It is thus highly desirable to zero the ballbot pitch and roll body angles before taking off. This is done with the leg mechanisms.

When all three legs are down and remain down, the three legs and body form an overconstrained spatial linkage [13]. The top view of ballbot with all three legs deployed is shown in Fig. 12. The spatial linkage consisting of the ballbot and the three legs attached to the floor with PR joints was simulated in Open Dynamics Engine. For each leg, the leg nut was moved up and the effect of leg nut position on the body angles (both roll and pitch) was recorded. In Fig. 12 it can be seen that leg 1 affects only the pitch (rotation about $y$ axis) and not the roll, while legs 2 and 3 affect both.

In an attempt to create a controller that would adjust the leg positions to tilt the body close to vertical, leg 1 was adjusted to the desired pitch and legs 2 and 3 were adjusted to the desired roll. (The mechanical design constrains the body tilt to a maximum of $5^{\circ}$.) A graph showing leg 1 position as a function of body pitch angle is shown in Fig. 13. Similar graphs hold for legs 2 and 3 . As can be seen, the relationship between leg position $\xi$ and body angle $\phi$ is approximately linear of the form $\xi=K_{\text {leg }} \phi+c_{\text {leg }}$. This relationship was used to create a PID controller that adjusts the leg positions so as to tilt the body to a desired roll and pitch angle as shown in Fig. 14. This controller facilitates a smooth auto transition from SSS to DSS as the initial body angles can be adjusted to be very close to zero. Transitioning from DSS to SSS is done by turning off the balancing controller when

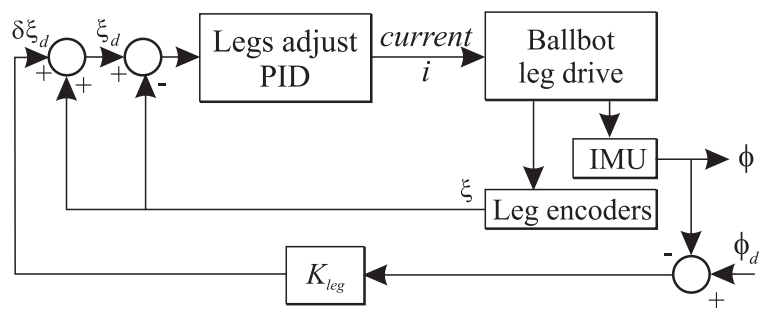

Fig. 14. Block diagram for the legs-adjust controller.

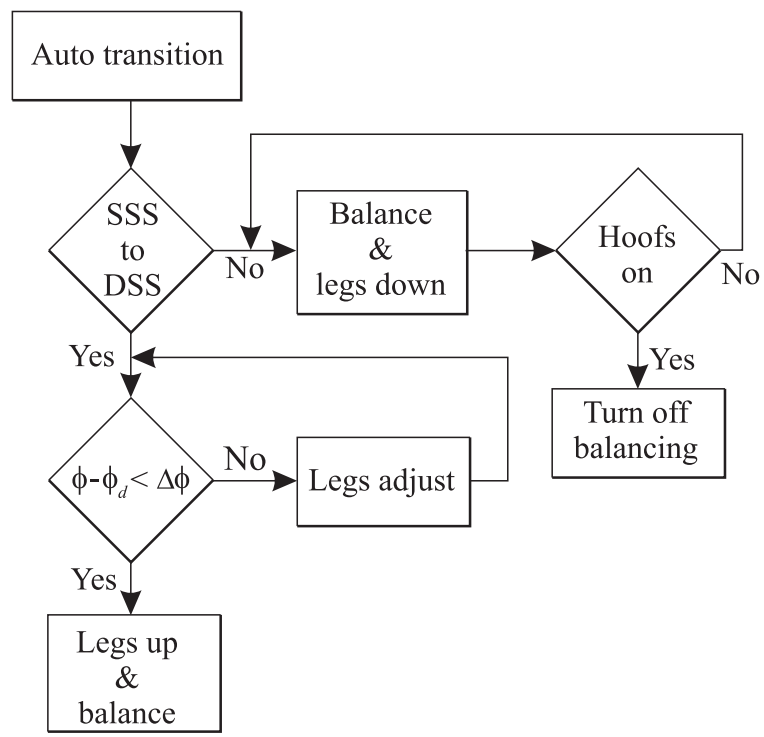

Fig. 15. Flow chart for the auto transition operation.

the hoof switches on the legs contact the floor. A flow chart is given in Fig. 15.

\section{RESULTS}

The balancing controller described in Sec. V was used to balance the ballbot. The variation of body pitch angle with time during the balancing operation is approximately $\pm 0.05^{\circ}$ as shown in Fig. 16(a). Similar results were obtained for roll. The result of a $360^{\circ}$ yaw rotation while station keeping is shown in Fig. 16(b). Fig. 17 shows selected frames of the $360^{\circ}$ yaw motion while balancing. The results show the capability of the robot to rotate in place, which in the future will be needed when the ballbot uses arms to manipulate objects.

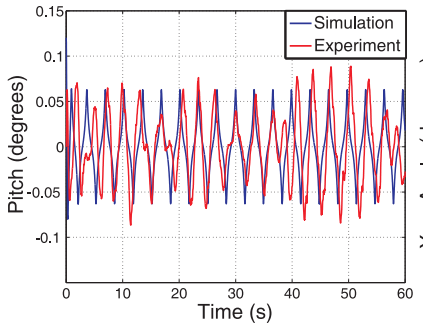

(a)

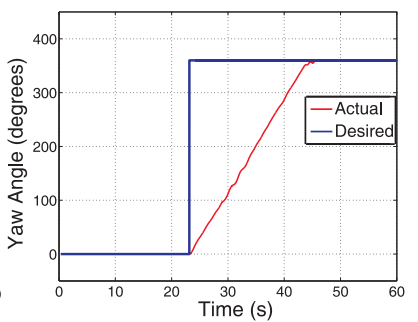

(b)
Fig. 16. Performance vs. time: (a) pitch axis during balancing, (b) $360^{\circ}$ yaw maneuver while balancing. 


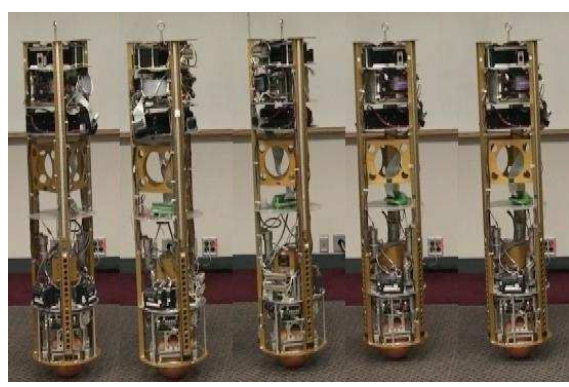

Fig. 17. Selected frames of $360^{\circ}$ yaw motion video.

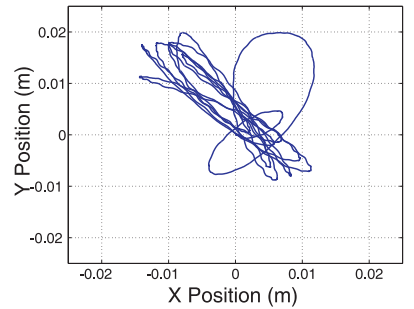

(a)

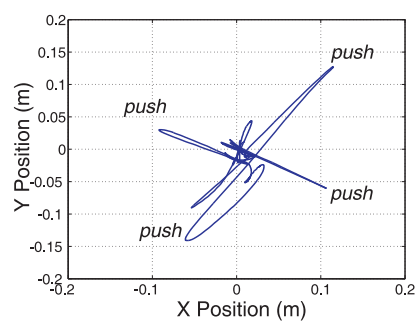

(b)
Fig. 18. Balancing at a position: (a) ball track on the carpeted floor using only the balancing controller, $(b)$ operation of the station keeping controller when the body is pushed off its position.

Fig. 18(a) is an XY plot of the ball position on a carpeted floor. As one can see, the balancing controller is able to keep the ball close to its starting point on floor to within about $\pm 10-15 \mathrm{~mm}$. Unlike the balancing controller, the station keeping controller described in Sec. V keeps the ball close to its starting point even when disturbed. Results of when the body is pushed by the hand in all four directions is shown in Fig. 18(b).

Finally, Fig. 19 shows selected frames of automatic transition from SSS to DSS and vice versa, taken from a companion video "Dynamically Stable Single-Wheeled Mobile Robot: Ballbot.”

\section{CONCLUSION}

A balancing controller was designed to stabilize the single spherical wheeled dynamically stable mobile robot. A station keeping controller was designed as a wrapper around the balancing controller to enable balancing at a fixed location in the presence of external disturbances. These controllers were found to be more robust than previously implemented

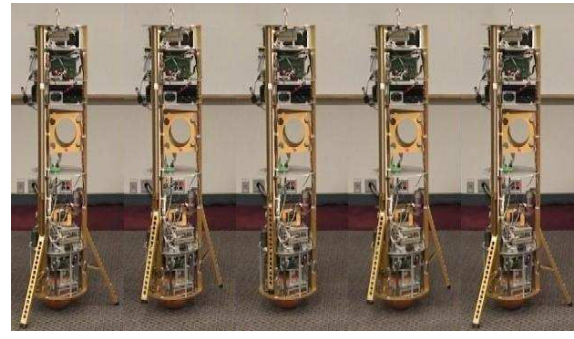

Fig. 19. Selected frames of automatic transition from SSS to DSS and vice versa.
LQR controllers. Many off-line experiments were performed to find numerical values for the ballbot's physical characteristics. Controllers for the yaw and leg drive mechanisms were designed and implemented. A control procedure for automatic transition from statically stable state (SSS) to dynamically stable state (DSS) and vice versa was designed and tested on the ballbot. The implementation of the controllers and their successful and robust operation on the ballbot has been demonstrated.

The work to date opens up a wide range of possibilities for ballbot's actions. The authors have ongoing research on motion planning and control for ballbot which is discussed in a companion paper [11]. With these considerations, it is reasonable to believe the ballbot represents a new class of wheeled mobile robots capable of agile, omni-directional motion.

\section{ACKNOWLEDGEMENTS}

The authors wish to thank Eric Schearer, Kathryn Rivard, Suresh Nidhiry, Jun Xian Leong, Jared Goerner, and Tom Lauwers for their superb efforts on the ballbot project.

\section{REFERENCES}

[1] Y.-S. Ha and S. Yuta. Indoor navigation of an inverse pendulum type autonomous mobile robot with adaptive stabilization control system. In Experimental Robotics IV, 4th Int'l. Symp., pages 529-37, 1997.

[2] H. G. Nguyen, J. Morrell, K. Mullens, A. Burmeister, S. Miles, N. Farrington, K. Thomas, and D. Gage. Segway robotic mobility platform. In SPIE Proc. 5609: Mobile Robots XVII, Philadelphia, PA, October 2004.

[3] P. Deegan, B. Thibodeau, and R. Grupen. Designing a self-stabilizing robot for dynamic mobile manipulation. Robotics: Science and Systems - Workshop on Manipulation for Human Environments, 2006.

[4] Tom Lauwers, George Kantor, and Ralph Hollis. One is enough! In Proc. Int'l. Symp. for Robotics Research, San Francisco, October 1215 2005. Int'l. Foundation for Robotics Research.

[5] T. B. Lauwers, G. A. Kantor, and R. L. Hollis. A dynamically stable single-wheeled mobile robot with inverse mouse-ball drive. In Proc. Int'l. Conf. on Robotics and Automation, Orlando, FL, May 15-19 2006.

[6] Masaaki Kumagai and Takaya Ochiai. Development of a robot balancing on a ball. International Conference on Control, Automation and Systems, 2008.

[7] László Havasi. ERROSphere: an equilibrator robot. International Conference on Control and Automation (ICCA2005), pages 971-976, June 27-29 2005.

[8] H. Wang and et al. An experimental method for measuring the moment of inertia of an electric power wheelchair. Proc. 29th Annual Int'l. Conf. of IEEE EMB, pages 4798-4801, 2007.

[9] R. Kelly, J. Llamas, and R. Campa. A measurement procedure for viscous and coulomb friction. IEEE Transactions on Instrumentation and Measurement, 49(4):857-861, 2000.

[10] Umashankar Nagarajan, George Kantor, and Ralph Hollis. Trajectory planning and control of an underactuated dynamically stable single spherical wheeled mobile robot. Proc. IEEE Int'l. Conf. on Robotics and Automation, May 2009.

[11] A.K. Mampetta. Automatic transition of ballbot from statically stable state to dynamically stable state. Master's thesis, Carnegie Mellon University, Pittsburgh, PA, 2006. Report CMU-RI-TR-01-00.

[12] S. Tsai, E. Ferreira, and C. Paredis. Control of the gyrover: A singlewheel gyroscopically stabilized robot. In IEEE/RSJ Int'l Conf. on Intelligent Robots and Systems (IROS'99), October 1999. 